\title{
Improvement of Dysgeusia by Polaprezinc, a Zinc-L-carnosine, in Outpatients Receiving Cancer Chemotherapy
}

\author{
HIRONORI FUJII ${ }^{1}$, CHIEMI HIROSE ${ }^{1}$, MASASHI ISHIHARA ${ }^{1}$, HIROTOSHI IIHARA ${ }^{1}$, \\ HISASHI IMAI ${ }^{2}$, YOSHIHIRO TANAKA ${ }^{2}$, NOBUHISA MATSUHASHI ${ }^{2}$, TAKAO TAKAHASHI ${ }^{2}$, \\ KAZUYA YAMAGUCHI ${ }^{2}$, KAZUHIRO YOSHIDA ${ }^{2}$ and AKIO SUZUKI ${ }^{1}$ \\ ${ }^{1}$ Department of Pharmacy, Gifu University Hospital, Gifu, Japan; \\ ${ }^{2}$ Department of Surgical Oncology, Gifu University Graduate School of Medicine, Gifu, Japan
}

\begin{abstract}
Background/Aim: Dysgeusia is one of the adverse events frequently affecting patients undergoing cancer chemotherapy. Dysgeusia-induced anorexia could decrease patient's quality of life. The present study was designed to determine whether the zinc-containing compound polaprezinc improves chemotherapy-induced dysgeusia. Patients and Methods: The incidence of grade 2 dysgeusia was assessed in 634 patients receiving cancer chemotherapy in outpatient settings during January 2013 and June 2017. Polaprezinc was administered to patients showing grade 2 dysgeusia and the effect was compared with that in patients subjected to followup observation. Results: Grade 2 dysgeusia appeared in 80 patients $(12.6 \%)$, in whom pancreatic cancer and treatment with fluoropyrimidines were significant risks for dysgeusia. Polaprezinc, when administered to patients with grade 2 dysgeusia, significantly shortened the duration of dysgeusia compared with that in the follow-up observation group. Subgroup analysis indicated that polaprezinc was less effective in patients with pancreatic cancer, those receiving gemcitabine, or those whose age was 65 year-old and over. Conclusion: Chemotherapy-induced dysgeusia occurred with high frequency in patients with pancreatic cancer or in those receiving fluoropyrimidines. Polaprezinc was highly effective in improving the symptom of dysgeusia, except for patients with pancreatic cancer, those receiving gemcitabine and the elderly.
\end{abstract}

Dysgeusia is one of the adverse events that occurs frequently during cancer chemotherapy. The occurrence of dysgeusia

This article is freely accessible online.

Correspondence to: Hironori Fujii, Department of Pharmacy, Gifu University Hospital, Yanagido 1-1, Gifu 501-1194, Japan. Tel: +81 582307080, Fax: +81 582307093, e-mail: h_fujii@gifu-u.ac.jp

Key Words: Dysgeusia, cancer chemotherapy, pancreatic cancer, fluoropyrimidines, gemcitabine, polaprezinc. associated with cancer chemotherapy is reported to appear approximately in $67 \%$ of all cases and in $38 \%$ of moderate to severe cases (1-2). According to the Common Terminology Criteria for Adverse Events (CTCAE) version 4.0 (3), dysgeusia is graded into two categories, grade 1 and grade 2, and is not life-threatening, thus the symptom is often overlooked in clinical practice. However, the disturbance of taste deprives patients of the enjoyment of food and causes nutritional derangement due to the reduction in appetite, which leads to impairment of patient's quality of life (4-6). Moreover, in lung cancer patients receiving crizotinib, anorexia associated with dysgeusia led to the discontinuation of therapy due to the loss of body weight (7). Therefore, chemotherapy-induced dysgeusia should be carefully managed for the maintenance of patient's quality of life and continuation of cancer therapy.

Although the precise mechanisms underlying chemotherapyinduced dysgeusia remain to be clarified, inhibition of the differentiation and proliferation of cells in the taste buds by the direct toxic action of chemotherapy drugs and the deficiency in zinc due to chelation by some chemotherapeutic agents may be implicated in the etiology of dysgeusia (8). In general, cells with a rapid turnover, including those in the taste buds, are more susceptible to anticancer drugs than other cells (9). Moreover, it has been demonstrated that a high amount of zinc is required for the regeneration of cells in taste buds, thereby indicating that zinc deficiency may cause the impairment of gustatory sense (10). Fukasawa et al. (11) reported that 5-fluorouracil has the ability to chelate zinc ions, which may contribute, at least in part, to the induction of dysgeusia (12). Thus, in addition to 5fluorouracil's direct toxic action on mucosal cells of the taste buds, deficiency in zinc appears to contribute to the gustatory dysfunction associated with this drug. Therefore, supplementation of zinc is likely to be effective for alleviating dysgeusia. However, there have only been few studies on the effect of zinc supplementation on dysgeusia. Yamagata et al. (13) showed in a preliminary study composed of a small number of patients receiving cancer chemotherapy, that premedication with zinc reduces the incidence of dysgeusia. 
Table I. Comparison of demographic between patients showing grade 2 dysgeusia and those without significant dysgeusia.

\begin{tabular}{|c|c|c|c|c|c|}
\hline & \multicolumn{2}{|c|}{ With dysgeusia $(\mathrm{N}=80)$} & \multicolumn{2}{|c|}{ Without dysgeusia $(\mathrm{N}=554)$} & $p$-Value \\
\hline Age (average, range) & \multicolumn{2}{|c|}{$66.3(22-83)$} & \multicolumn{2}{|c|}{$65.9(22-90)$} & $0.590^{\mathrm{a}}$ \\
\hline Gender (male/female) & \multicolumn{2}{|c|}{$41 / 39$} & \multicolumn{2}{|c|}{$276 / 278$} & $0.811^{\mathrm{b}}$ \\
\hline Height $(\mathrm{cm})$ & \multicolumn{2}{|c|}{$160.4 \pm 7.5$} & \multicolumn{2}{|c|}{$159.7 \pm 53.0$} & $0.515^{\mathrm{c}}$ \\
\hline Body weight $(\mathrm{kg})$ & \multicolumn{2}{|c|}{$54.6 \pm 9.1$} & \multicolumn{2}{|c|}{$55.6 \pm 18.8$} & $0.376^{\mathrm{c}}$ \\
\hline Body mass index & \multicolumn{2}{|c|}{$21.2 \pm 3.3$} & \multicolumn{2}{|c|}{$21.7 \pm 10.4$} & $0.254^{\mathrm{c}}$ \\
\hline Serum creatinine $(\mathrm{mg} / \mathrm{dl})$ & \multicolumn{2}{|c|}{$0.73 \pm 0.24$} & \multicolumn{2}{|c|}{$0.70 \pm 0.25$} & $0.463^{\mathrm{c}}$ \\
\hline Creatinine clearance $(\mathrm{ml} / \mathrm{min})$ & \multicolumn{2}{|c|}{$77.10 \pm 25.50$} & \multicolumn{2}{|c|}{$81.30 \pm 29.90$} & $0.384^{\mathrm{c}}$ \\
\hline Cancer type & $\mathrm{N}$ & $\%$ & $\mathrm{~N}$ & $\%$ & \\
\hline Colorectal cancer & 29 & 36.3 & 195 & 35.2 & $0.854^{\mathrm{b}}$ \\
\hline Pancreatic cancer & 20 & 25.0 & 78 & 14.1 & $0.012^{\mathrm{b}}$ \\
\hline Gastric cancer & 12 & 15.0 & 51 & 9.2 & $0.105^{\mathrm{b}}$ \\
\hline Breast cancer & 6 & 7.5 & 54 & 9.7 & $0.521^{\mathrm{b}}$ \\
\hline Malignant lymphoma & 3 & 3.8 & 54 & 9.7 & $0.080^{\mathrm{b}}$ \\
\hline Lung cancer & 4 & 5.0 & 43 & 7.8 & $0.378^{\mathrm{b}}$ \\
\hline Others & 6 & 7.5 & 79 & 14.3 & $0.097^{\mathrm{b}}$ \\
\hline Chemotherapy drugs & $\mathrm{N}$ & $\%$ & $\mathrm{~N}$ & $\%$ & \\
\hline Fluoropyrimidines & 48 & 60.0 & 267 & 48.2 & $0.048^{\mathrm{b}}$ \\
\hline Gemcitabine & 18 & 22.5 & 83 & 15.0 & $0.086^{\mathrm{b}}$ \\
\hline Taxanes & 11 & 13.8 & 92 & 16.6 & $0.517^{\mathrm{b}}$ \\
\hline Platinum & 40 & 50.0 & 295 & 53.2 & $0.586^{\mathrm{b}}$ \\
\hline Anthracyclines & 7 & 8.8 & 81 & 14.6 & $0.156^{\mathrm{b}}$ \\
\hline
\end{tabular}

aMann-Whitney $U$-test; ${ }^{\mathrm{b}}$ chi-square test; ${ }^{\mathrm{c}}$-test.

On the other hand, in patients with idiopathic taste impairment with zinc deficiency, oral administration of polaprezinc (zinc-L-carnosine) has been reported to improve taste disturbance by elevating plasma zinc concentration. We previously reported, that pretreatment with polaprezinc at a daily oral dose of $75 \mathrm{mg}$ prevents the occurrence of dysgeusia associated with radiochemotherapy in patients with head and neck cancer (14).

Therefore, in the present study, the incidence and risk of dysgeusia were investigated in patients who received cancer chemotherapy in our outpatient chemotherapy clinic. The effect of oral administration of polaprezinc on the recovery from dysgeusia was subsequently examined in patients who showed grade 2 symptoms, and the effect was compared with that obtained from patients who were subjected to follow-up observation.

\section{Patients and Methods}

Patients. A total of 1,098 patients received cancer chemotherapy in our outpatient chemotherapy clinic during the period between Jan 2013 and Jun 2017. Among them, 464 patients had received cancer chemotherapy, thus, the remaining 634 patients who did not have cancer chemotherapy were the subjects included in the present study. Data obtained from the electronic medical records of our hospital were analyzed retrospectively.

The present study was carried out in accordance with the guidelines for human studies adopted by the ethics committee of the Gifu University Graduate School of Medicine, and notified by the Japanese government (Institutional review board approval No.26156). In view of the retrospective nature of the study, the need for informed consent from subjects was not mandated.

Assessment of dysgeusia. Dysgeusia was graded according to the Common Terminology Criteria for Adverse Events (CTCAE) version 4.0 (3): grade 1 is characterized by altered taste but no change in diet, and grade 2 is characterized by altered taste with change in diet (e.g., oral supplements), noxious or unpleasant taste, and loss of taste.

Risk analysis for dysgeusia. Demographics of patients who received cancer chemotherapy were compared between patients who experienced grade 2 dysgeusia and those who did not. Factors that showed significant differences between the two groups were subjected to univariate and multivariate logistic regression analyses.

Evaluation of the effect of polaprezinc. Eighty patients who showed grade 2 dysgeusia were orally administered with polaprezinc at a daily dose of $150 \mathrm{mg}$ (twice a day) until the symptom disappeared, while the follow-up observation group did not receive treatment 
Fujii et al: Polaprezinc and Dysgeusia Caused by Chemotherapy

Table II. Uni- and multi-variate logistic regression analyses for the risk of grade 2 dysgeusia in outpatients receiving cancer chemotherapy.

\begin{tabular}{|c|c|c|c|c|c|c|}
\hline & \multicolumn{3}{|c|}{ Univariate analysis } & \multicolumn{3}{|c|}{ Multivariate analysis } \\
\hline & OR & $(95 \% \mathrm{CI})$ & $p$-Value & OR & $(95 \% \mathrm{CI})$ & $p$-Value \\
\hline Pancreatic cancer & 2.034 & $(1.162-3.561)$ & 0.013 & 4.467 & $(1.024-19.491)$ & 0.046 \\
\hline Gastric cancer & 1.740 & $(0.884-3.428)$ & 0.109 & 1.545 & $(0.747-3.197)$ & 0.241 \\
\hline Use of gemcitabine & 1.647 & $(0.928-2.926)$ & 0.088 & 0.657 & $(0.135-3.190)$ & 0.603 \\
\hline Use of fluoropyrimidines & 1.612 & $(1.000-2.599)$ & 0.050 & 1.999 & $(1.101-3.632)$ & 0.023 \\
\hline
\end{tabular}

except for azulene gargle solution. Kaplan-Meier plots of the time course of grade 2 symptoms were compared between the polaprezinc group and follow-up observation group.

Subgroup analysis for the effect of polaprezinc. The odds ratio (OR) and $95 \%$ confidence interval (CI) for the effect of polaprezinc were calculated with respect to the demographic characteristics of patients.

Statistical analyses. Data were analyzed using IBM SPSS version 22 (IBM Japan Ltd., Tokyo, Japan) and GraphPad Prism version 6.0 (GraphPad Software, San Diego, CA, USA). p-Values less than 0.05 were considered significant. In order to compare the demographics of patients between the two groups, $t$-test was used for parametric variables and chi-square test or Mann-Whitney $U$-test was used for non-parametric variables. The incidence rates of grade 2 dysgeusia were compared by Kruskal-Wallis test followed by Scheffe's test for multiple comparison. For comparison of the incidence of dysgeusia between the two groups, chi-square test or Fisher's exact probability test was carried out. Kaplan-Meier estimation was used to analyze the improvement of grade 2 dysgeusia and median time to recovery from grade 2 dysgeusia was compared by Mantel-Cox log rank test.

\section{Results}

Comparison of demographics between patients with and without grade 2 dysgeusia. The incidence rate of dysgeusia was $41.8 \%$ (268/634) for all grades and $12.6 \%(80 / 634)$ for grade 2 . To determine the risk of grade 2 dysgeusia, demographics of patients were compared between patients who had grade 2 dysgeusia and those who did not. As shown in Table I, pancreatic cancer $(25.0 \%$ vs. $14.1 \%, p=0.012)$ and fluoropyrimidines (60.0\% vs. $48.2 \%, p=0.048)$ were more prevalent in patients who experienced grade 2 dysgeusia than in those who did not. Other factors, including age, gender, height, body weight, renal function, cancer types other than pancreatic cancer, and chemotherapy drugs excluding fluoropyrimidines, were not significantly different between the two groups.

Risk factors for dysgeusia. As shown in Table II, a multivariate logistic regression analysis showed that pancreatic cancer $(\mathrm{OR}=4.467 ; 95 \% \mathrm{CI}=1.024-19.491 ; p=0.046)$ and use of fluoropyrimidines $(\mathrm{OR}=1.999 ; 95 \% \mathrm{CI}=1.101-3.632$; $p=0.023$ ) were significant risk factors for grade 2 dysgeusia. Interestingly, the incidence rate of grade 2 dysgeusia was elevated as the number of risk factors increased: $7.3 \%$ for no risk factors, $14.9 \%$ ( $p=0.021$ versus no risk) for one risk factor and $41.7 \%$ ( $p=0.002$ versus no risk) for two risk factors.

Improvement of grade 2 dysgeusia by oral administration of polaprezinc. The rate of recovery from grade 2 dysgeusia within 90 days after the onset of symptoms was $60.0 \%$ (24 of 40 patients) in patients who administered oral polaprezinc, which was significantly higher than that $(22.5 \%, 9$ of 40 patients) in the follow-up observation group ( $\mathrm{OR}=5.167$; 95\%CI=1.94-3.700; $p=0.0007)$. As shown in Figure 1, Kaplan-Meier plots indicated that the median time to recovery from grade 2 dysgeusia was significantly shorter in the polaprezinc group than in the follow-up observation group (63 days versus 112 days, hazard ratio (HR), 1.778; $95 \% \mathrm{CI}=1.275-2.280 ; p=0.019)$.

As shown in Table III, there were no significant differences in the demographics of patients, except for age, in which median age was significantly $(p=0.028)$ lower in the polaprezinc group than in the follow-up observation group.

Subgroup analysis of the action of polaprezinc. The OR for the curative effect of polaprezinc compared to the follow-up observation was evaluated with respect to the demographics of patients. As shown in Figure 2, polaprezinc was less effective $(\mathrm{OR}=1.89 ; 95 \% \mathrm{CI}=0.41-8.61 ; p=0.662)$ in the elderly (65 years old and older) than in patients younger than 65 years old $(\mathrm{OR}=5.50 ; 95 \% \mathrm{CI}=1.63-18.55 ; \quad p=0.011)$. Patients with pancreatic cancer were less responsive to polaprezinc $(\mathrm{OR}=1.40$; $95 \% \mathrm{CI}=0.20-10.03 ; p=0.867)$ than those with other types of cancer. Moreover, use of gemcitabine interfered with the effect of polaprezinc $(\mathrm{OR}=0.88 ; 95 \% \mathrm{CI}=0.11-7.11 ; p=0.685)$. In contrast, patients with colorectal cancer had the highest response to polaprezinc $(\mathrm{OR}=20.0 ; 95 \% \mathrm{CI}=2.05-195.0 ; p=0.008)$.

\section{Discussion}

In the present study, the incidence rate of dysgeusia was $41.8 \%$ for all grades and $12.6 \%$ for grade 2 in patients who received cancer chemotherapy in our outpatient chemotherapy clinic. Hovan et al. (15) showed in a 
systematic review of 26 clinical studies that the incidence rates of all grades of dysgeusia were 56-76\%, depending on the type of cancer treatment. However, our data were generally consistent with the data reported by Ishikawa et al. (16) who showed that dysgeusia occurs in $43.8 \%$ of outpatients receiving cancer chemotherapy. Similar data were also shown by Sugawara et al. (17), who reported that taste disorder appears in $45.9 \%$ of patients who undergo cancer chemotherapy in outpatient settings. On the other hand, Perrone et al. (18) reported a less frequent incidence of dysgeusia in breast cancer patients treated with docetaxel (23\% for grade 1 and $2 \%$ for grade 2 ). Therefore, it is considered that the incidence of chemotherapy-induced dysgeusia varies among different chemotherapeutic regimens.

Imai et al. (12) reported that the chemotherapeutic regimens containing 5-fluorouracil cause dysgeusia more frequently than those without 5-fluorouracil (48.1\% vs. $38.8 \%$ ). Sugawara et al. (17) also showed an increase in the incidence of dysgeusia by the addition of 5-fluorouracil. Interestingly, our multivariate logistic regression analysis showed that the use of fluoropyrimidines was a significant risk factor for grade 2 dysgeusia. Fluoropyrimidines cause injury of the oral mucosa (19) as well as the gustatory nerve (20). Moreover, these compounds inhibit the absorption of zinc ions by inducing their chelation (11). All of these fluoropyrimidine actions may contribute to the development of dysgeusia.

On the other hand, we reported here for the first time that pancreatic cancer was a significant risk factor for grade 2 dysgeusia. Zinc is absorbed from the upper digestive tract after associating with metallothionein, a zinc-binding protein that is present abundantly in the pancreatic juice (21-22). In addition, insulin promotes the transition of zinc from the lumen to the epithelium of the intestinal tract. Yu et al. (23) reported a deficiency of zinc in patients with pancreatoduodenectomy and the recovery from zinc deficiency by supplementation of pancreatic enzymes. In the present study, partial pancreatectomy was performed in the majority of patients with pancreatic cancer. Taken together, it is suggested that the decrease in the absorption of zinc due to the reduction in metallothionein in pancreatic cancer patients with partial pancreatic resection contributes, at least in part, to the high incidence of dysgeusia.

Although various compounds, including zinc compounds (13, 24-26), amifostine $(15,27)$, cannabinoids (28) and vitamin D (29), have been tested for the purpose of preventing or treating chemotherapy-induced dysgeusia, most of them have shown limited effect.

We previously reported that oral administration of polaprezinc at a daily dose of $75 \mathrm{mg}$ in patients who received radiochemotherapy for head and neck cancer, is more effective than placebo for the prevention of grade 2 taste
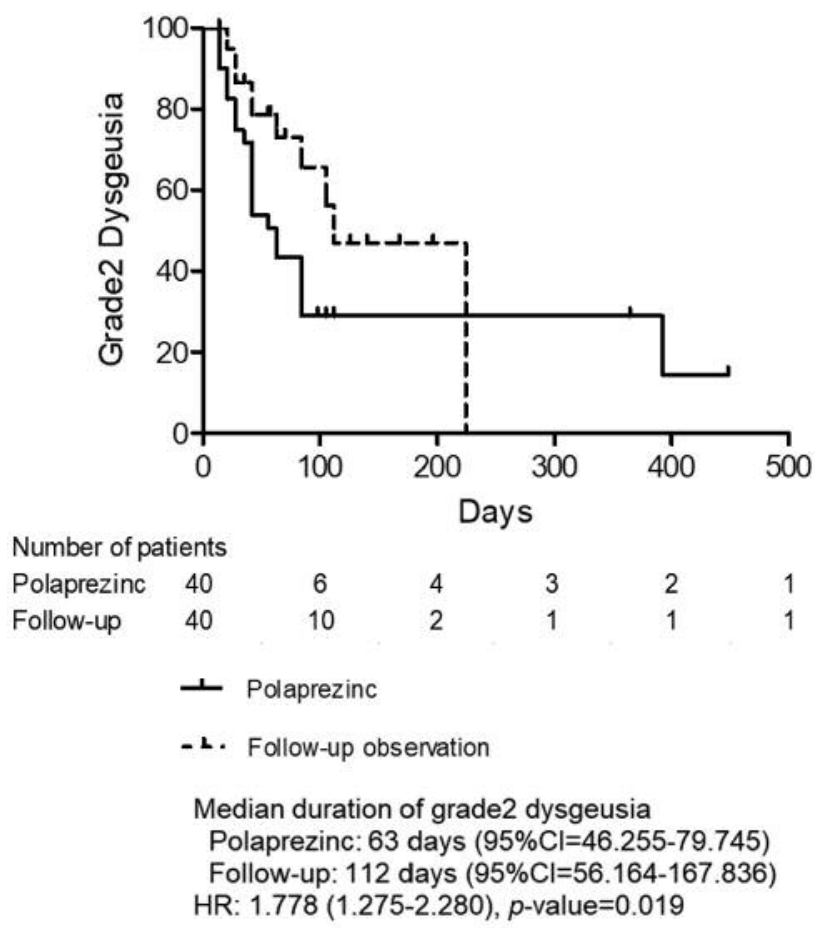

Figure 1. Kaplan-Meier plots comparing the effects of polaprezinc and follow-up observation on the duration of grade 2 dysgeusia in outpatients receiving cancer chemotherapy. Polaprezinc was administered to 40 patients showing grade 2 dysgeusia, while follow-up observation was carried out to the remaining 40 patients showing grade 2 dysgeusia. Median duration was statistically compared by Mantel-Cox log-rank test.

disturbance $(6.7 \%$ vs. $53.3 \%, p=0.006)$ and grade $>2$ mucositis (40.0\% vs. $86.7 \%, p=0.009)$ (14). Sakagami et al. (30) reported that polaprezinc increases serum zinc level dose-dependently and also improves gustatory dysfunction in patients with idiopathic taste disorder.

Polaprezinc (Promac granules $15 \%{ }^{\circledR}$, Zeria Pharmaceutical Co., Ltd, Tokyo, Japan) is zinc-L-carnosine that has been approved in Japan as an anti-ulcer drug with gastric mucosal protective action. This drug contains $34 \mathrm{mg}$ zinc in one $\mathrm{g}$ of Promac granule preparation and the dosage for gastric ulceration is half a $\mathrm{g}$ of the preparation that contains $75 \mathrm{mg}$ polaprezinc or $17 \mathrm{mg}$ zinc twice in a day.

In the present study, patients with grade 2 dysgeusia either received polaprezinc at a daily dose of $150 \mathrm{mg}$ or were subjected to follow-up observation. As a result, significant improvement of taste disturbance was observed in the polaprezinc group. Kaplan-Meier estimates also indicated that the median time to recovery from grade 2 dysgeusia was significantly shorter in the polaprezinc group than in the follow-up observation group $(\mathrm{HR}=1.778$; 95\% CI=1.2752.280; $p=0.019$ ). 


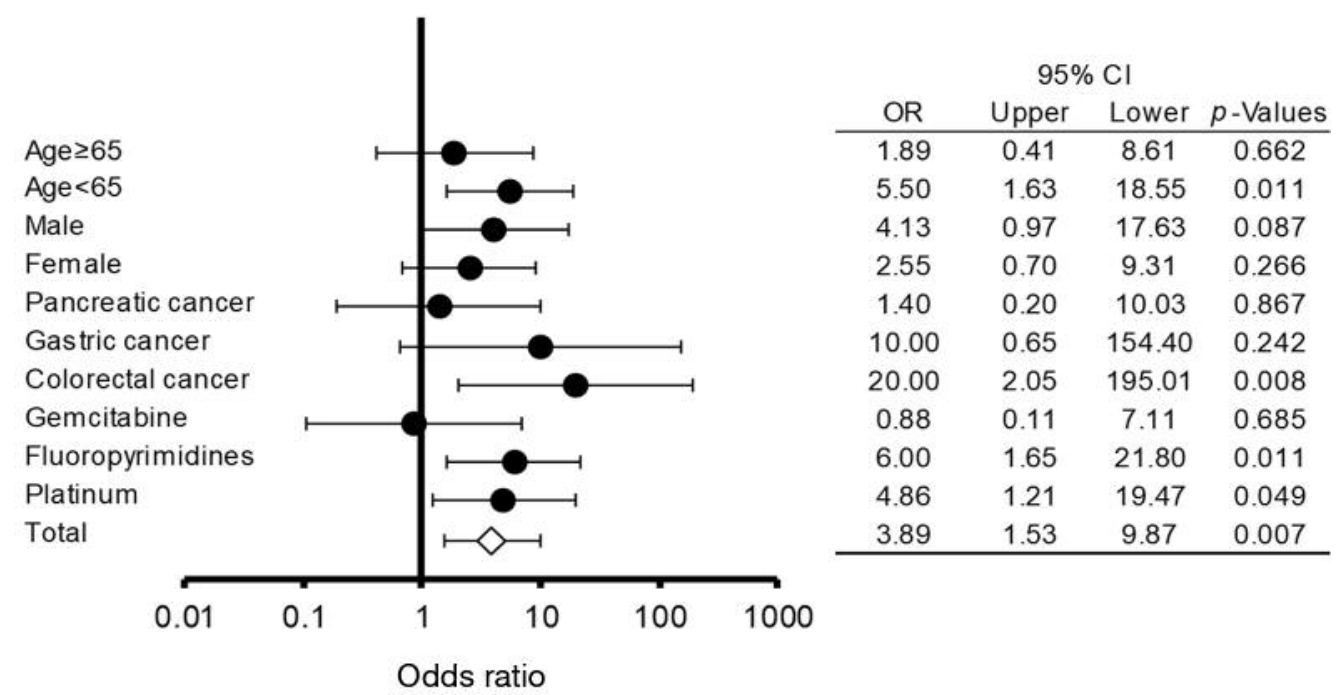

Figure 2. Subgroup analysis for the improving action of polaprezinc against chemotherapy-induced dysgeusia in patients who showed grade 2 dysgeusia. OR for the relief of dysgeusia induced by polaprezinc to that obtained by the follow-up observation was assessed in each patient group. Data were statistically analyzed by chi-square test.

Table III. Comparison of demographics of patients receiving polaprezinc or those subjected to follow-up observation after appearance of grade 2 dysgeusia.

\begin{tabular}{|c|c|c|c|c|c|}
\hline & \multicolumn{2}{|c|}{ With polaprezinc $(\mathrm{N}=40)$} & \multicolumn{2}{|c|}{ Without polaprezinc $(\mathrm{N}=40)$} & $p$-Value \\
\hline Age (average, range) & \multicolumn{2}{|c|}{$64.0(22-81)$} & \multicolumn{2}{|c|}{$68.6(39-83)$} & $0.028^{\mathrm{a}}$ \\
\hline Gender (male/female) & \multicolumn{2}{|c|}{$19 / 21$} & \multicolumn{2}{|c|}{$22 / 18$} & $0.502^{\mathrm{b}}$ \\
\hline Height $(\mathrm{cm})$ & 161.0 & 6.9 & 159.8 & 8.0 & $0.378^{\mathrm{c}}$ \\
\hline Body weight (kg) & 54.6 & 8.7 & 54.6 & 9.5 & $0.911^{\mathrm{c}}$ \\
\hline Body mass index & 21.0 & 2.9 & 21.4 & 3.6 & $0.088^{\mathrm{c}}$ \\
\hline Serum creatinine $(\mathrm{mg} / \mathrm{dl})$ & 0.70 & 0.24 & 0.75 & 0.25 & $0.796^{\mathrm{c}}$ \\
\hline Creatinine clearance $(\mathrm{ml} / \mathrm{min})$ & 82.55 & 26.84 & 71.69 & 23.17 & $0.338^{\mathrm{c}}$ \\
\hline Cancer type & $\mathrm{N}$ & $\%$ & $\mathrm{~N}$ & $\%$ & \\
\hline Colorectal cancer & 17 & 42.5 & 12 & 30.0 & $0.245^{\mathrm{b}}$ \\
\hline Pancreatic cancer & 8 & 20.0 & 12 & 30.0 & $0.439^{\mathrm{b}}$ \\
\hline Gastric cancer & 3 & 7.5 & 9 & 22.5 & $0.115^{\mathrm{b}}$ \\
\hline Breast cancer & 3 & 7.5 & 3 & 7.5 & $1.000^{\mathrm{b}}$ \\
\hline Others & 7 & 17.5 & 6 & 15.0 & $1.000^{\mathrm{b}}$ \\
\hline Chemotherapy drugs & $\mathrm{N}$ & $\%$ & $\mathrm{~N}$ & $\%$ & \\
\hline Fluorouracil & 22 & 55.0 & 26 & 65.0 & $0.361^{\mathrm{b}}$ \\
\hline Gemcitabine & 9 & 22.5 & 9 & 22.5 & $1.000^{\mathrm{b}}$ \\
\hline Taxanes & 4 & 10.0 & 7 & 17.5 & $0.518^{\mathrm{b}}$ \\
\hline Platinum & 21 & 52.5 & 19 & 47.5 & $0.655^{\mathrm{b}}$ \\
\hline Anthracyclines & 3 & 7.5 & 4 & 10.0 & $1.000^{\mathrm{b}}$ \\
\hline
\end{tabular}

${ }^{\mathrm{a}}$ Mann-Whitney $U$-test; ${ }^{\mathrm{b}} \mathrm{Chi}$-square test; ${ }^{\mathrm{c}} \mathrm{t}$-test.

Subgroup analysis indicated that polaprezinc was less effective in the elderly (over 65 years old), in patients with pancreatic cancer, or in those who received gemcitabine. The lower susceptibility to polaprezinc in the elderly may be explained by a possible reduction in the absorption of zinc.
On the other hand, zinc absorption might be lowered in pancreatic cancer patients with partial resection of pancreas due to the reduction in metallothionein. Therefore, it is likely that supplementation of zinc by the use of polaprezinc is not sufficient to elevate the level of zinc, which might be one of 
the reasons why the effect of polaprezinc was not sufficient in pancreatic cancer patients. Thus, supplementation of pancreatic enzymes in addition to polaprezinc seemed to be required for the effective amelioration of dysgeusia in patients with pancreatic cancer.

In addition, most of the patients with pancreatic cancer received gemcitabine in the present study, which may explain why the use of gemcitabine interfered with the effect of polaplezinc. In contrast, polaprezinc was highly effective in improving dysgeusia in patients with colorectal cancer who received platinum or fluoropyrimidines.

There were several limitations of the present study: It was a single center, retrospective study, there were no zinc concentration measurements, and the relation between the development of dysgeusia and blood zinc concentration was not adequately assessed.

In conclusion, pancreatic cancer and the use of fluoropyrimidines were significant risk factors for grade 2 dysgeusia in outpatients receiving cancer chemotherapy. Oral administration of polaprezinc at a daily dose of $150 \mathrm{mg}$ was effective in ameliorating grade 2 dysgeusia, although the compound was less effective in patients with pancreatic cancer, those who received gemcitabine and the elderly.

\section{Conflicts of Interest}

K. Yoshida has received grants, personal fees, and nonfinancial support from Chugai Pharmaceutical Co., Ltd. during the conduction of this study; grants and personal fees from Taiho Pharmaceutical Co., Ltd.; grants and personal fees from Pfizer Inc.; grants and personal fees from Yakult Honsha Co., Ltd.; grants from Bristol-Myers Squibb; grants from Kyowa Hakko Kirin Co., Ltd., outside the submitted work; and honoraria from Taiho Pharmaceutical Co., Ltd., Pfizer Inc., Chugai Pharmaceutical Co., Ltd., Kyowa Hakko Kirin Co., Ltd., and Yakult Honsha Co., Ltd.; and had a consultant or advisory relationship with Taiho Pharmaceutical Co., Ltd. and La Roche, Ltd. T. Takahashi has received honoraria for lectures from Takeda Pharmaceutical Co., Ltd. The other Authors have no conflicts of interest.

\section{References}

1 Bernhardson BM, Tishelman C and Rutqvist LE: Self-reported taste and smell changes during cancer chemotherapy. Support Care Cancer 16: 275-283, 2008.

2 Wickham RS, Rehwaldt M, Kefer C, Shott S, Abbas K, GlynnTucker E, Potter C and Blendowski C: Taste changes experienced by patients receiving chemotherapy. Oncol Nurs Forum 26: 697-706, 1999.

3 U.S. Department of Health and Human Services, National Institutes of Health National Cancer Institute (2009) Common Terminology Criteria for Adverse Events (CTCAE) Version 4.0. (https://www.eortc.be/services/doc/ctc/).

4 Holmes S: Food avoidance in patients undergoing cancer chemotherapy. Support Care Cancer 1: 326-330, 1993.

5 Boltong A, Keast R and Aranda S: Experiences and consequences of altered taste, flavour and food hedonics during chemotherapy treatment. Support Care Cancer 20: 2765-2774, 2012.
6 Epstein JB and Barasch A: Taste disorders in cancer patients: pathogenesis, and approach to assessment and management. Oral Oncol 46: 77-81, 2010.

7 Koizumi T, Fukushima T, Tatai T, Kobayashi T, Sekiguchi N, Sakamoto A and Sasaki S: Successful treatment of crizotinibinduced dysgeusia by switching to alectinib in ALK-positive non-small cell lung cancer. Lung Cancer 88: 112-113, 2015.

8 Kawaguchi M, Sawaki K, Okubo M, Sakai T, Shinomiya T and Kosuge Y: Adverse drug reactions and oral disorders. Nihon Yakurigaku Zasshi 127: 447-453, 2006.

9 Comeau TB, Epstein JB and Migas C: Taste and smell dysfunction in patients receiving chemotherapy: a review of current knowledge. Support Care Cancer 9: 575-580, 2001.

10 Henkin RI and Bradley DF: Hypogeusia corrected by $\mathrm{Ni}^{++}$and $\mathrm{Zn}^{++}$. Life Sci II 9: 701-709, 1970.

11 Fukasawa T, Orii T, Tanaka M, Yano S, Suzuki N and Kanzaki Y: Statistical approach to drug-induced taste disorders based on zinc chelating ability. Nippon Yakugaku Zasshi 125: 377-387, 2005.

12 Imai $H$, Soeda $H$, Komine $K$, Otsuka $K$ and Shibata $H$ : Preliminary estimation of the prevalence of chemotherapyinduced dysgeusia in Japanese patients with cancer. BMC Palliat Care 12: 38, 2013.

13 Yamagata T, Nakamura Y, Yamagata Y, Nakanishi M, Matsunaga K, Nakanishi H, Nishimoto T, Minakata Y, Mune M and Yukawa $\mathrm{S}$ : The pilot trial of the prevention of the increase in electrical taste thresholds by zinc containing fluid infusion during chemotherapy to treat primary lung cancer. J Exp Clin Cancer Res 22: 557-563, 2003.

14 Watanabe T, Ishihara M, Matsuura K, Mizuta K and Itoh Y: Polaprezinc prevents oral mucositis associated with radiochemotherapy in patients with head and neck cancer. Int $\mathrm{J}$ Cancer 127: 1984-1990, 2010.

15 Hovan AJ, Williams PM, Stevenson-Moore P, Wahlin YB, Ohrn KE, Elting LS, Spijkervet FK and Brennan MT; Dysgeusia Section, Oral Care Study Group, Multinational Association of Supportive Care in Cancer (MASCC)/International Society of Oral Oncology (ISOO): A systematic review of dysgeusia induced by cancer therapies. Support Care Cancer 18: 10811087, 2010.

16 Ishikawa T, Morita J, Kawachi K and Tagashira H: Incidence of dysgeusia associated with chemotherapy for cancer. Gan To Kagaku Ryoho 40: 1049-1054, 2013.

17 Sugawara S, Takimoto N, Iida A, Mori K, Sugiura M, Yamamura $\mathrm{K}$ and Adachi $\mathrm{M}$ : Incidence of taste disorder associated with cancer patients undergoing chemotherapy on an ambulatory basis. Gan To Kagaku Ryoho 36: 1871-1876, 2009.

18 Perrone F, Nuzzo F, Di Rella F, Gravina A, Iodice G, Labonia V, Landi G, Pacilio C, Rossi E, De Laurentiis M, D'Aiuto M, Botti G, Forestieri V, Lauria R, De Placido S, Tinessa V, Daniele B, Gori S, Colantuoni G, Barni S, Riccardi F, De Maio E, Montanino A, Morabito A, Daniele G, Di Maio M, Piccirillo MC, Signoriello S, Gallo C and de Matteis A: Weekly docetaxel versus $\mathrm{CMF}$ as adjuvant chemotherapy for older women with early breast cancer: final results of the randomized phase III ELDA trial. Ann Oncol 26: 675-682, 2015.

19 Jones JA, Avritscher EB, Cooksley CD, Michelet M, Bekele BN and Elting LS: Epidemiology of treatment-associated mucosal injury after treatment with newer regimens for lymphoma, breast, lung, or colorectal cancer. Support Care Cancer 14: 505$515,2006$. 
20 Aoki K, Obata K, Kurihara M, Kuniyasu H, Kirita T and Takaki M: Possible peripheral mechanism for taste disorder in rats administered S-1. Int J Clin Oncol 19: 549-556, 2014.

21 De Lisle RC, Sarras MP Jr., Hidalgo J and Andrews GK: Metallothionein is a component of exocrine pancreas secretion: implications for zinc homeostasis. Am J Physiol 271: C1103$1110,1996$.

22 Lee DK, Geiser J, Dufner-Beattie J and Andrews GK: Pancreatic metallothionein-I may play a role in zinc homeostasis during maternal dietary zinc deficiency in mice. J Nutr 133: 45-50, 2003.

$23 \mathrm{Yu}$ HH, Yang TM, Shan YS and Lin PW: Zinc deficiency in patients undergoing pancreatoduodenectomy for periampullary tumors is associated with pancreatic exocrine insufficiency World J Surg 35: 2110-2117, 2011.

24 Halyard MY, Jatoi A, Sloan JA, Bearden JD 3rd, Vora SA, Atherton PJ, Perez EA, Soori G, Zalduendo AC, Zhu A, Stella PJ and Loprinzi CL: Does zinc sulfate prevent therapy-induced taste alterations in head and neck cancer patients? Results of phase III double-blind, placebo-controlled trial from the North Central Cancer Treatment Group (N01C4). Int J Radiat Oncol Biol Phys 67: 1318-1322, 2007.

25 Halyard MY: Taste and smell alterations in cancer patients--real problems with few solutions. J Support Oncol 7: 68-69,2009.

26 Najafizade N, Hemati S, Gookizade A, Berjis N, Hashemi M, Vejdani S, Ghannadi A, Shahsanaee A and Arbab N: Preventive effects of zinc sulfate on taste alterations in patients under irradiation for head and neck cancers: A randomized placebocontrolled trial. J Res Med Sci 18: 123-126, 2013.
27 Komaki R, Lee JS, Milas L, Lee HK, Fossella FV, Herbst RS, Allen PK, Liao Z, Stevens CW, Lu C, Zinner RG, Papadimitrakopoulou VA, Kies MS, Blumenschein GR Jr, Pisters KM, Glisson BS, Kurie J, Kaplan B, Garza VP, Mooring D, Tucker SL and Cox JD: Effects of amifostine on acute toxicity from concurrent chemotherapy and radiotherapy for inoperable non-small-cell lung cancer: report of a randomized comparative trial. Int J Radiat Oncol Biol Phys 58: 1369-1377, 2004.

28 Davis MP: Cannabinoids for symptom management and cancer therapy: the evidence. J Natl Compr Canc Netw 14: 915-922, 2016.

29 Fink M: Vitamin D deficiency is a cofactor of chemotherapyinduced mucocutaneous toxicity and dysgeusia. J Clin Oncol 29: e81-82, 2011.

30 Sakagami M, Ikeda M, Tomita H, Ikui A, Aiba T, Takeda N, Inokuchi A, Kurono Y, Nakashima M, Shibasaki Y and Yotsuya O: A zinc-containing compound, polaprezinc, is effective for patients with taste disorders: randomized, double-blind, placebo-controlled, multi-center study. Acta Otolaryngol 129: 1115-1120, 2009.

Received September 12, 2018

Revised October 1, 2018

Accepted October 3, 2018 